archives-ouvertes

\title{
Resistance of Aedes aegypti (Diptera: Culicidae) Populations to Deltamethrin, Permethrin, and Temephos in Cambodia
}

Sébastien Boyer, Sergio Lopes, Didot Prasetyo, John Hustedt, Ay Sarady, Dyna Doum, Sony Yean, Borin Peng, Sam Bunleng, Rithea Leang, et al.

\section{To cite this version:}

Sébastien Boyer, Sergio Lopes, Didot Prasetyo, John Hustedt, Ay Sarady, et al.. Resistance of Aedes aegypti (Diptera: Culicidae) Populations to Deltamethrin, Permethrin, and Temephos in Cambodia. Asia-Pacific Journal of Public Health, SAGE Publications, 2018, 30, pp.158 - 166. 10.1177/1010539517753876 . hal-03054020

\section{HAL Id: hal-03054020 \\ https://hal.archives-ouvertes.fr/hal-03054020}

Submitted on 11 Dec 2020

HAL is a multi-disciplinary open access archive for the deposit and dissemination of scientific research documents, whether they are published or not. The documents may come from teaching and research institutions in France or abroad, or from public or private research centers.
L'archive ouverte pluridisciplinaire HAL, est destinée au dépôt et à la diffusion de documents scientifiques de niveau recherche, publiés ou non, émanant des établissements d'enseignement et de recherche français ou étrangers, des laboratoires publics ou privés. 


\title{
Resistance of Aedes aegypti \\ (Diptera: Culicidae) Populations \\ to Deltamethrin, Permethrin, and \\ Temephos in Cambodia
}

\author{
Sébastien Boyer, $\mathbf{P h D}^{1}\left(\mathbb{D}\right.$, Sergio Lopes, $\mathrm{Msc}^{2}$, \\ Didot Prasetyo, $\mathrm{PhD}^{3}$, John Hustedt, Msc', \\ Ay Sao Sarady, Msc², Dyna Doum, Msc², Sony Yean, Msc', \\ Borin Peng, Msc', Sam Bunleng, Msc ${ }^{4}$, Rithea Leang, PhD ${ }^{4}$, \\ Didier Fontenille, $\mathrm{PhD}^{\prime}$, and Jeffrey $\mathrm{Hii}, \mathrm{PhD}^{5}$
}

\begin{abstract}
Dengue fever is a major public health concern, including 185,000 annual cases in Cambodia. Aedes aegypti is the primary vector for dengue transmission and is targeted with insecticide treatments. This study characterized the insecticide resistance status of Ae aegypti from rural and urban locations. The susceptibility to temephos, permethrin, and deltamethrin of $\mathrm{Ae}$ aegypti was evaluated in accordance with World Health Organization instructions. All the field populations showed lower mortality rate to temephos compared with the sensitive strain with resistance ratio $50\left(R R_{50}\right)$ varying from 3.3 to 33.78 and $R R_{90}$ from 4.2 to 47 compared with the sensitive strain, demonstrating a generalized resistance of larvae to the temephos in Cambodia. Ae aegypti adult populations were highly resistant to permethrin regardless of province or rural/ urban classification with an average mortality of $0.02 \%$. Seven of the 8 field populations showed resistance to deltamethrin. These results are alarming for dengue vector control, as widespread resistance may compromise the entomological impact of larval control operations. Innovative vector control tools are needed to replace ineffective pesticides in Cambodia.
\end{abstract}

\section{Keywords}

Aedes aegypti, Cambodia, insecticide, mosquito, resistance, vector control

\section{Introduction}

Dengue fever is a major public health concern, with estimates of 400 million cases every year in urban, suburban, and rural tropical areas. ${ }^{1}$ In Cambodia, around 185,000 cases are estimated

\footnotetext{
'Institut Pasteur du Cambodge, Phnom Penh, Cambodia. 2Malaria Consortium Cambodia, Phnom Penh, Cambodia

${ }^{3}$ US Naval Medical Research Unit-2 Detachment Phnom Penh, Phnom Penh, Cambodia ${ }^{4}$ National Center for Entomology, Parasitology and Malaria Control (CNM), Phnom Penh, Cambodia

${ }^{5}$ Malaria Consortium Asia Regional Office, Faculty of Tropical Medicine, Mahidol University Bangkok, Thailand

Corresponding Author:

Sébastien Boyer, Medical Entomology Platform, Institut Pasteur du Cambodge, 5 Boulevard Monivong, Phnom Penh, Cambodia.

Email: sboyer@pasteur-kh.org
} 
annually. ${ }^{2}$ The primary vector for dengue transmission is Aedes aegypti, which favors environments where water storage is abundant and solid waste disposal is deficient. ${ }^{3}$ As Ae aegypti is implicated in the transmission of arboviruses such as Zika, chikungunya, and yellow fever, ${ }^{4}$ vector control strategies that target Ae aegypti populations may have an major public health impact. Many insecticides have been used in order to control Ae aegypti populations, but little information exists on the susceptibility of Cambodian populations to the most commonly used insecticides.

As early as 1955, DDT (dichlorodiphenyltrichloroethane) residual spray was used in the first malaria eradication pilot in Snuol district. ${ }^{5}$ DDT was again used in public health programs targeting malaria and dengue in urban and rural areas and at UNHCR refugee camps along the Cambodia-Thailand border from 1981 to 1987, after which it was no longer imported. ${ }^{6}$ Pyrethroids, particularly permethrin and deltamethrin, were introduced to Cambodia in the late 1980s and 2000 for the control of malaria (impregnation of bednets) and dengue (thermal fogging and ULV [ultra-low volume] sprays), respectively. ${ }^{6}$ Since 1992, temephos has been imported with roughly 200 tons per year used mainly for larval control of dengue vectors. ${ }^{6}$ In 1966 , Mouchet and $\mathrm{Chastel}^{7}$ showed total susceptibility of Ae aegypti to DDT, fenthion, malathion, and diazinon insecticides, but observed resistance to dieldrin and $\gamma$-HCH ( $\gamma$-hexachlorocyclohexane). More recently, Ae aegypti resistance to temephos was also investigated during 2 field studies in Cambodia. ${ }^{8}$ The resistance pattern and future of temephos is increasingly important as this larvicide has been the main dengue control strategy used by National Dengue Control Program (NDCP) for more than 20 years and for biannual larvicide campaigns since 2001. 3,6

Using the World Health Organization (WHO) diagnostic dose $(0.02 \mathrm{mg} / \mathrm{L})$, the Phnom Penh population tested in 2001 was found to be resistant to temephos, while Kampong Cham population was still susceptible. More recently, among 7 Ae aegypti populations, 6 were found to be resistant to temephos with mortality ranging from $11.02 \%$ up to $88.62 \%$ at the WHO diagnostic concentration (To Setha, personal communication). While it seems clear that temephos resistance among Ae aegypti populations has increased over time in Cambodia, the patterns between rural and urban areas are as delineated.

While pyrethroid and organophosphate insecticides are used in the national malaria and dengue control programs, significant use of insecticides (including larvicides, repellents, space sprays, treated materials, and coils) at home and in the private sector results in unquantifiable use of insecticides. Coupled with the lack of information on adult resistance status in Cambodia and long-term usage of space spraying by pest control companies and public health authorities, the need for characterizing the susceptibility of Ae aegypti to pyrethroids is urgent. This study aims to characterize the insecticide resistance status for immature and adult stages of Ae aegypti collected from rural and urban Cambodian environment. Eight field populations were tested using WHO test procedures against the most commonly used insecticides in Cambodia, which include temephos (for immature stages) and deltamethrin/permethrin (for adult stages).

\section{Material and Methods}

\section{Mosquito Collection}

Four different geographical areas in Cambodia were selected for field sample collections (Phnom Penh, Kampong Cham, Battambang, and Siem Reap). Two urban villages and 2 rural villages were selected as collection points within each village. Villages were selected by NDCP according to geographical representation, dengue incidence, and recent use of temephos (within the previous 2 years) (Supplementary File 1, available in the online version of the article). Twenty-five households were randomly selected within each village and all containers were inspected for larvae and pupae using direct pipetting for small containers and sweep net method for large 
containers. ${ }^{9}$ Collected larvae/pupae were pooled by location (rural/urban) in each province and transported to an insectary.

Larvae and pupae were reared in standard conditions (temperature, $28^{\circ} \mathrm{C} \pm 1{ }^{\circ} \mathrm{C}$; relative humidity, $75 \% \pm 2.5 \%$; photoperiod, 12 hours day/night) in $24.8 \times 19.7 \times 3.8 \mathrm{~cm}$ standard white plastic larval tray containing $2 \mathrm{~L}$ of purified water and fed with half a teaspoon of grounded fish food daily until adult emergence. Adult Aedes were separated from other species by direct aspiration and each population was separated by location (total of 8 populations from 4 provinces).

For both larvae and adult assays, a USDA (United States Department of Agriculture) reference susceptible strain ${ }^{10}$ was used as positive and negative control with water and ethanol in plastic beakers.

\section{Rearing of FI Larvae for Testing}

Adult Aedes mosquitos from parental generations were reared at standard conditions and fed with $10 \%$ sucrose solution. All populations were also provided with lab-reared mice for blood meal once every 3 days for 3 to 4 hours. Eggs from the F1 generation were collected on white filter paper and placed inside black plastic cups. Eggs were dried and stored in envelops and later sent to the laboratory. F1 eggs were immersed in water according to assay needs for testing procedures and larvae were reared as previously described.

\section{Aedes aegypti Larval Bioassays}

In accordance with WHO instructions, ${ }^{11}$ late third instar larvae of F1 generation were used for determining the resistance of mosquito larvae to temephos.

Temephos (Sigma, Pestanal analytical grade, $250 \mathrm{mg}$ ) was diluted in ethanol to produce a stock solution of $1000 \mathrm{mg} / \mathrm{L}$. The main stock solution was diluted into several working concentrations better suited for testing. All solutions were stored in glass bottles and labeled accordingly. To obtain each of these concentrations the adequate volume of temephos was pipetted from stock solutions, adding the remaining amount of solution with ethanol into each beaker containing $99 \mathrm{~mL}$ of water. Four replicates were used for every concentration, and each replicate consists of 25 larvae.

Six temephos concentrations $(0.2,0.05,0.03,0.02,0.01,0.004 \mathrm{mg} / \mathrm{L})$ were used to determine lethal concentration (LC) 50/95 (eg, the necessary concentrations needed to kill $50 \% / 95 \%$ of mosquito larvae). Resistance ratios $\left(\mathrm{RR}_{50}\right.$ and $\left.\mathrm{RR}_{95}\right)$ were calculated dividing $\mathrm{LC}_{50}$ and $\mathrm{LC}_{95}$ rates from Ae aegypti field populations by the $\mathrm{LC}_{50}$ and $\mathrm{LC}_{90}$ rates of the USDA susceptible strain.

\section{Ae. aegypti Adult Bioassays}

Insecticide resistance screening for adult mosquitos was conducted using the WHO tube assay. ${ }^{11}$ Two synthetic pyrethroids; permethrin and deltamethrin, at diagnostic concentrations appropriate for Aedes mosquitoes were used. WHO tube kit and impregnated permethrin $(0.25 \%)$, deltamethrin $(0.03 \%)$, and piperonyl butoxide for synergist assay (PBO $4 \%$ ) papers were obtained from Vector Control Research Unit at the University of Science, Penang, Malaysia. Diagnostic and synergist concentrations were chosen following WHO recommendations. ${ }^{11}$

For this bioassay, each tested population used 4 tubes containing permethrin $(0.25 \%), 4$ tubes containing deltamethrin $(0.03 \%)$, and 4 control tubes containing silicone oil paper. Twenty-five adults at least 3 days old and non-blood-fed female mosquitoes were introduced into each tube lined with untreated paper (holding tube) for 60 minutes. Mosquitoes were then transferred into the exposure tube and exposed to impregnated paper for 60 minutes. Mosquito knock down (KD) was measured at the end of the exposure, after which mosquitoes were transferred back to the 
Table I. Mean Lethal Concentration $(\mathrm{LC})_{50}$ and $\mathrm{LC}_{90}( \pm \mathrm{SE})$ of 8 Aedes aegypti Larval Populations With Temephos in Cambodia. ${ }^{a}$

\begin{tabular}{llcrcr}
\hline Environment & Populations $^{\mathrm{b}}$ & $\mathrm{LC}_{50}(\mathrm{SE})$ & $\mathrm{RR}_{50}$ & $\mathrm{LC}_{90}(\mathrm{SE})$ & $\mathrm{RR}_{90}$ \\
\hline Urban & Phnom Penh & $0.020(0.0006)$ & 5.4 & $0.028(0.0008)$ & 6.0 \\
& Siem Reap & $0.014(0.0008)$ & 3.8 & $0.020(0.0008)$ & 4.2 \\
& Kampong Cham & $0.031(0.0012)$ & 8.4 & $0.052(0.0025)$ & 11.1 \\
& Battambang & $0.125(0.0044)$ & 33.8 & $0.221(0.0082)$ & 47.0 \\
\multirow{4}{*}{ Rural } & Phnom Penh & $0.014(0.0007)$ & 3.8 & $0.031(0.0011)$ & 6.6 \\
& Siem Reap & $0.012(0.0006)$ & 3.3 & $0.021(0.0010)$ & 4.4 \\
& Kampong Cham & $0.048(0.0015)$ & 13.0 & $0.066(0.0029)$ & 14.0 \\
& Battambang & $0.041(0.0015)$ & 11.1 & $0.064(0.0031)$ & 13.6 \\
\hline
\end{tabular}

${ }^{a} R_{50}$ and $R R_{90}$ represent the resistance ratio of the field populations compared with the US Department of Agriculture (USDA) susceptible reference strain.

bUSDA strain: $\mathrm{LC}_{50}=0.0037 \pm 0.00008 \mathrm{mg} / \mathrm{L} ; \mathrm{LC}_{90}=0.0047 \pm 0.000 \mathrm{I} \mathrm{mg} / \mathrm{L}$.

tube without insecticide. Mortality was counted at the end of a 24-hour period and the resistance status was interpreted according to the WHO protocol.

Insecticide-synergist assay using $\mathrm{PBO}$ was conducted to measure the effect of preexposure to a synergist on the expression of insecticide resistance. Adult Aedes were preexposed to this synergist for 1 hour before exposure to insecticide. KD and mortality were recorded the same way as standard tests.

\section{Data Management and Statistical Analysis}

$\mathrm{KD}$ and mortality were registered at 1 and 24 hours postexposure, respectively. RRs for larvae and adult mosquitos were calculated by dividing the average mortality found in each field population by the mortality obtained with the USDA susceptible reference strain.

For larvae results, $\mathrm{LC}_{50}$ and $\mathrm{LC}_{90}$ were obtained by plotting the mortality using log probit analysis.

Statistical analysis (analysis of variance and mean comparison) were completed to compare the mortality of adults to permethrin and deltamethrin with or without the use of PBO. Graphs and data analysis were done with R software. ${ }^{12}$

\section{Results}

\section{Larval Bioassays}

The overall bioassay results for larvae are presented in Table 1 . The highest $\mathrm{LC}_{50}$ and $\mathrm{LC}_{90}$ values were obtained with Battambang urban populations $\left(\mathrm{LC}_{50}=0.125 \pm 0.004 \mathrm{mg} / \mathrm{L}\right.$ and $\mathrm{LC}_{90}=0.221 \pm 0.008 \mathrm{mg} / \mathrm{L}$ ) and Kampong Cham (Table 1). In Phnom Penh and Siem Reap, the $\mathrm{LC}_{50}$ and $\mathrm{LC}_{90}$ were lowest with $\mathrm{LC}_{50}$ values ranging between $0.012 \mathrm{mg} / \mathrm{L}$ (Siem Reap rural) and $0.020 \mathrm{mg} / \mathrm{L}$ (Phnom Penh rural).

The RR for urban and rural populations of Siem Reap and Phnom Penh provinces were mostly above the threshold, which is defined as a resistant population with $R R \geq 5$. $R R$ values of Kampong Cham and Battambang urban and rural populations were 2- and 9-fold higher than the threshold, respectively. While these results may be linked to the continued distribution of temephos and consequent exposure of populations to this chemical, it is of great concern that 2 out of 4 populations in these 2 provinces registered RRs twice as high as the defined resistance threshold (Kampong Cham Rural, $\mathrm{RR}=13.0$; Battambang rural, $\mathrm{RR}=11.2$ ) and 1 province registered an RR 6 times higher than the defined threshold (Battambang urban, $R R=33.6$ ). 


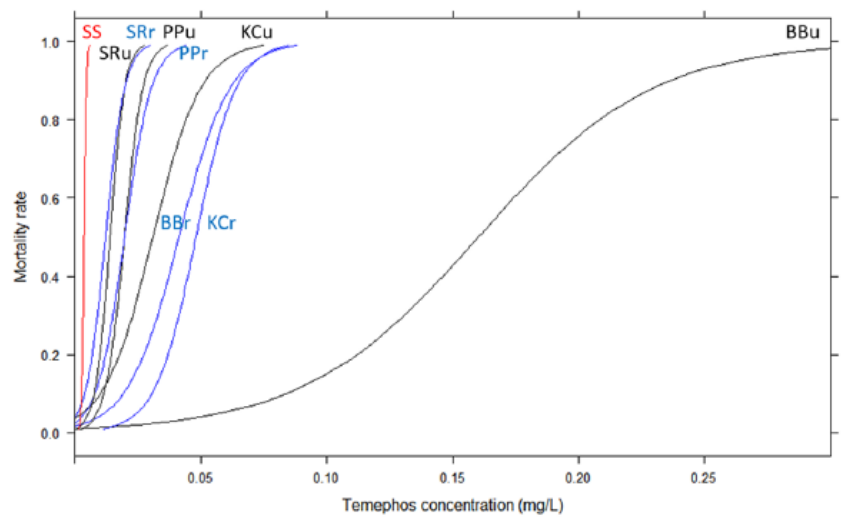

Figure I. Mortality rate of Aedes aegypti larvae to tempehos in the 4 provinces. The 4 urban populations are represented in black, the rural populations in blue. The red line is the sensitive strain (SS). BB, Battambang; KC, Kampong Chan; SR, Siem Reap; PP, Phnom Penh. The lowercase letters "r" and " $\mathrm{u}$ " represent rural and urban areas, respectively.

Higher lethal doses $\left(\mathrm{LC}_{50}\right.$ or $\left.\mathrm{LC}_{90}\right)$ are needed to kill Ae. aegypti larvae from Battambang and Kampong Cham populations as depicted on the four mortality curves on the right side of the graph compared to Siem Reap and Phnom Penh populations (Figure 1). Finally, all the field populations showed higher mortality curve patterns compared with the sensitive strain over a range of concentrations (Figure 1).

\section{Adult Bioassays}

Results showed a very high level of resistance to permethrin regardless of province or rural/urban classification (Figure 2; Supplementary File 2, available in the online version of the article). The average mortality to permethrin at the WHO diagnostic dose is $2.22 \% \pm 0.02 \%$ for all the populations. While all populations showed resistance to permethrin, six of the eight populations showed no mortality to permethrin at all. The additional 2 Kampong Cham populations had $1.1 \%$ and $3.9 \%$ of mortality. Adult bioassays showed a significant difference in mortality to permethrin depending on the population and the presence of PBO $(F=3.35 ; d f=8 ; P=.003)$, particularly a significant increase in mortality from $1.1 \%$ to $18.6 \%$ in rural population from Kampong Cham province (Supplementary File 2).

Seven of the 8 field populations had a percentage less than $90 \%$ of mortality due to deltamethrin, meaning that these populations are resistant. The average mortality of Ae aegypti populations from Phnom Penh and Siem Reap provinces ranged between $4.0 \%$ and $8.3 \%$ only. A significant difference in mortality to deltamethrin among the five highest mortality populations $(>52 \%)$ tested were observed in the presence of $\mathrm{PBO}(F=7.20 ; d f=8 ; P<.0001)$.

\section{Discussion}

\section{Resistance to Temephos: Implications for Public Health}

Observed Ae aegypti resistance to temephos is consistent with a recent study where 6 of 7 populations showed similar resistance in Cambodia (To Setha, personal communication). The $\mathrm{RR}_{50}$ range of the 8 populations to temephos between 3.8 and 33.6 reflects the intensity of insecticide control. In Thailand, despite mosquito resistance to deltamethrin and permethrin, temephos is 


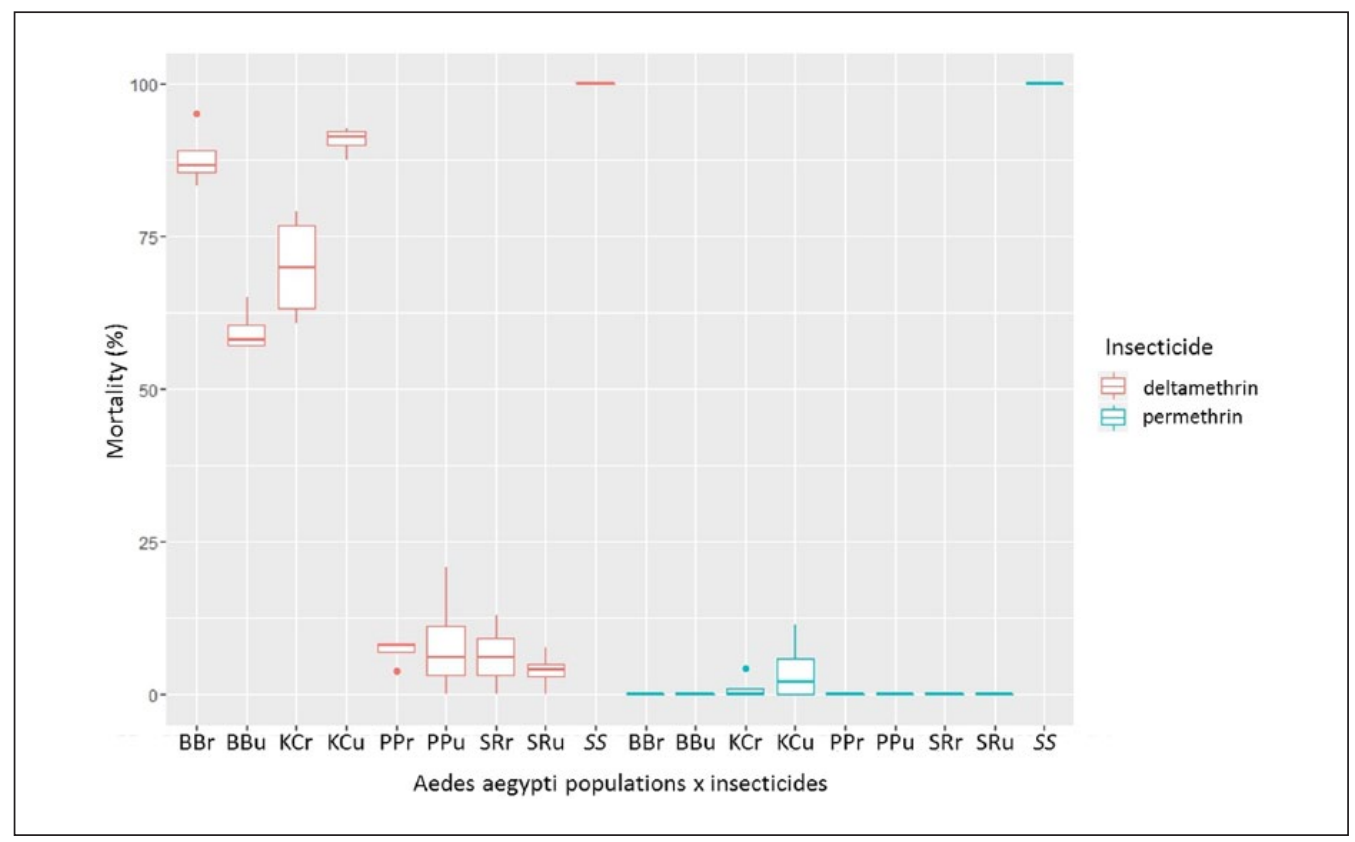

Figure 2. Mortality of Aedes aegypti populations to deltamethrin $(0.03 \%)$ and permethrin $(0.25 \%)$ following recommended WHO diagnostic doses. BB, Battambang; KC, Kampong Cham; PP, Phnom Penh; SR, Siem Reap; SS USDA sensitive strain. The lowercase letters " $r$ " and "u" represent rural and urban areas, respectively.

still an effective insecticide to control Ae aegypti larvae. ${ }^{13}$ On the basis of data showing temephos resistance in Phnom Penh over 17 years, ${ }^{8}$ a review of prevention and control strategies should be conducted and highlight the effects of reliance on a single method of control (eg, high levels of temephos use in Cambodia ${ }^{14}$ may compromise the entomological impact of larval control operations).

Bacillus thuringiensis var israelensis (Bti) was tested with success in 2005 around Phnom Penh. ${ }^{15}$ A new Bti strain AM65-52 was tested in 2016 against Ae aegypti field population from Kandal province that was resistant to temephos. Results showed a reduction in the number of pupae over 13 weeks, with an average $70 \%$ reduction during the first 8 weeks. ${ }^{16}$ The use of the Poecilia reticulate (guppy) fish to control Aedes populations in water storage was tested in 2008 and after 1 year, a $79 \%$ reduction in Aedes larvae in community was observed with a presence of guppies in only $57 \%$ of the containers. ${ }^{17}$ In 2008, a new formulation of pyriproxifen was tested in water containers against Ae aegypti in Phum Thmei near Phnom Penh. ${ }^{18}$ The study identified an inhibition of adult emergence in treated jars reaching $90 \%$ for 20 weeks, and remaining $>80 \%$ until the end of the study ( 34 weeks). In Kampong Cham Province in 2008 water jars were covered with long-lasting insecticide net Permanet 2.0 (insecticide $=$ deltamethrin) without significant reductions in mosquitoes, ${ }^{17}$ possibly explained by the strong resistance to delamethrin that we observed in Ae aegypti adults. A large-scale randomized trial comparing guppy and COMBI (Communication for Behavioral Impact) in Kampong Cham showed 92.5\% reduction in larvalpositive containers and $76 \%$ to $88 \%$ coverage with guppies after 1 year. A recently completed cluster randomized control trial showed that an integrated vector management approach using guppy fish (Poecilia reticulata), a new slow release pyriproxyfen matrix (Sumilarv 2MR), and community engagement through a clear COMBI strategy reduced indoor adult density roughly $50 \%$ as compared with the control arm. ${ }^{19}$ All these methods focused on key containers, especially 
water cement jars that produced approximately $95 \%$ of Ae aegypti larvae and pupae ${ }^{9}$ and should be considered in Cambodia as a cost-effective replacement of temephos.

\section{Resistant to Permethrin but Susceptible to Deltamethrin}

Ae aegypti deltamethrin-resistant populations have been described in different countries in Asia, ${ }^{20}$ Latin America, ${ }^{21}$ Africa, ${ }^{22}$ Oceania, ${ }^{23}$ and the Caribbean. ${ }^{24}$ In our study, Ae aegypti populations were either totally resistant to deltamethrin (with 2 populations exhibiting 0 mortality) or had tolerance patterns. Recently, the same pattern was observed in Thailand where $A e$ aegypti $\mathrm{F} 1$ females were susceptible to deltamethrin, but resistant to permethrin. ${ }^{13}$ A substantial geographic variation exist to pyrethroid resistance, with lower adult resistance levels in Asia, Africa, and the United States. However, there is 250 -fold resistance to deltamethrin in Thailand. ${ }^{25}$

In this study, an extremely strong resistance to permethrin was observed both with and without PBO, which seems to indicate that the resistance is already fixed. Comparatively, the result with deltamethrin and deltamethrin + PBO suggests the involvement of detoxifying enzymes. However, generally multiple resistance between pyrethroids are possible and it can be expected that there is a $k d r$ mutation for resistance in both insecticides. As the mechanisms of resistance between permethrin and DDT are expected to be the same, via a $k d r$ mutation, ${ }^{26}$ the already existing DDT resistance ${ }^{7}$ may explain the current fixed resistance observed with permethrin. There are several $k d r$ mutations common in Aedes species that synergize with each other when they are associated. ${ }^{27}$ Heterozygous V1016G, and F1534F and F1534C mutants were found in Thailand, ${ }^{28}$ and the same mutation was also described southern China with V1016G mutants. ${ }^{29}$ There is substantial variation in $k d r$ in the Southeast Asian region that has effects on resistance (arising from different combinations of 3 mutations - S989P, V1016G, and F1534C — in Ae aegypti). Although there are other mutations detected in Ae aegypti, they do not appear to have effect on resistance based on current evidence. For example, combinations of F1534, C1534C, V1016G, and S989P29 are present in Cambodia and may act together with metabolic resistance. The resistance patterns to deltamethrin and permethrin in the Cambodian villages fit with the variation in frequencies of the three mutations and especially in low 989/1016 but high 1534 in permethrin (but not deltamethrin) resistant locations, but higher 989/1016 in Phnom Penh and Siem Reap (perhaps in combination with 1534).

Our results question the resistance mechanisms. Indeed, the absence of correlation between permethrin and deltamethrin may involve different effects induced by type I pyrethroid (permethrin) and a pseudo pyrethroid (nonester pyrethroid; deltamethrin), and so different resistance mechanisms. ${ }^{30}$

\section{Limitations and Conclusion}

We acknowledge the lack of baseline data on temephos distribution in the villages sampled. While temephos distribution has been acknowledged as the main outbreak response tool in Cambodia, ${ }^{3}$ the timing and concentrations used in the villages sampled in this study were not discriminated. Hence, we cannot fully characterize the existing preconditions of each village in terms of previous larviciding activities, but temephos distribution is organized annually at a national and province scales. Likewise, pyrethroid based interventions like thermal fogging, long-lasting insecticide nets usage and pyrethroid-based aerosol spray use was not characterized during field collection, limiting the possibility to ascertain potential drivers for the resistance patterns registered.

Nevertheless, our results and those of neighboring countries are alarming. From a regional point of view, it seems essential to rapidly change control methods and replace temephos with 
another larvicide that remains to be determined. Finally, and perhaps most worrying, it seems that in the event of an epidemic the adulticides used in the Southeast Asia region are no longer effective. We must quickly find an alternative.

\section{Declaration of Conflicting Interests}

The author(s) declared no potential conflicts of interest with respect to the research, authorship, and/or publication of this article.

\section{Funding}

The author(s) disclosed receipt of the following financial support for the research, authorship, and/or publication of this article: This study was supported by UK Department for International Development (DFID) through the Programme Partnership Arrangement (Award number: 40097745).

\section{Supplemental Material}

The supplementary material for this article is available online.

\section{ORCID iD}

Sébastien Boyer (iD) https://orcid.org/0000-0002-2946-586X

\section{References}

1. Guzman A, Istúriz RE. Update on the global spread of dengue. Int J Antimicrob Agents. 2010;36(suppl 1):S40-S42. doi:10.1016/j.ijantimicag.2010.06.018.

2. Shepard DS, Undurraga EA, Halasa YA. Economic and disease burden of dengue in Southeast Asia. PLoS Negl Trop Dis. 2013;7:e2055. doi:10.1371/journal.pntd.0002055.

3. Huy R, Buchy P, Conan A, et al. National dengue surveillance in Cambodia 1980-2008: epidemiological and virological trends and the impact of vector control. Bull World Health Organ. 2010;88:650-657.

4. Christophers SR. Aedes aegypti (L) the Yellow Fever Mosquito: Its Life History, Bionomics and Structure. New York, NY: Cambridge University Press; 1960.

5. Brown A. Personal experiences in the malaria eradication campaign 1955-1962. J R Soc Med. 2002;95:154-156.

6. Ministry of Environment of Cambodia. National Profile on chemicals management in Cambodia. http://www.un.org/esa/dsd/dsd_aofw_ni/ni_pdfs/NationalReports/cambodia/Full_Report.pdf. Published December 2004. Accessed September 9, 2017.

7. Mouchet J, Chastel C. La résistance aux insecticides chez Aedes aegypti L et Aedes albopictus a PhnomPenh (Cambodge). http://horizon.documentation.ird.fr/exl-doc/pleins_textes/pleins_textes_5/b_ fdi_08-09/11102.pdf. Accessed September 9, 2017.

8. Polson KA, Curtis C, Chang MS, Olson JG, Chantha N, Rawlins SC. Susceptibility of two Cambodian population of Aedes aegypti mosquito larvae to temephos during 2001. Dengue Bull. 2001;25:79-83.

9. Seng CM, Setha T, Nealon J, Socheat D. Pupal sampling for Aedes aegypti (L) surveillance and potential stratification of dengue high-risk areas in Cambodia. Trop Med Int Health. 2009;14:1233-1240. doi:10.1111/j.1365-3156.2009.02368.x.

10. Kuno G. Early history of laboratory breeding of Aedes aegypti (Diptera: Culicidae) focusing on the origins and use of selected strains. J Med Entomol. 2010;47:957-971. doi:10.1603/ME10152.

11. World Health Organization. Monitoring and managing insecticide resistance in Aedes mosquito populations. Interim guidance for entomologists. http://apps.who.int/iris/bitstream/10665/204588/2/WHO_ ZIKV_VC_16.1_eng.pdf. Published March 8, 2016. Accessed September 9, 2017.

12. R Core Team. The R Project for statistical computing. https://www.R-project.org/. Accessed September 9, 2017.

13. Thongwat D, Bunchu N. Susceptibility to temephos, permethrin and deltamethrin of Aedes aegypti (Diptera: Culicidae) from Muang district, Phitsanulok Province, Thailand. Asian Pac J Trop Med. 2015;8:14-18. doi:10.1016/S1995-7645(14)60180-2. 
14. Khun S, Manderson LH. Abate distribution and dengue control in rural Cambodia. Acta Trop. 2007;101:139-146. doi:10.1016/j.actatropica.2007.01.002.

15. Setha T, Chantha N, Socheat D. Efficacy of Bacillus thuringiensis israelensis, VectoBac ${ }^{\circledR}$ WG and DT, formulations against dengue mosquito vectors in cement potable water jars in Cambodia. Southeast Asian J Trop Med Public Health. 2007;38:261-268.

16. Setha T, Chantha N, Benjamin S, Socheat D. Bacterial Larvicide, Bacillus thuringiensis israelensis strain AM 65-52 water dispersible granule formulation impacts both dengue vector, Aedes aegypti (L) population density and disease transmission in Cambodia. PLoS Negl Trop Dis. 2016;10:e0004973. doi:10.1371/journal.pntd.0004973.

17. Seng CM, Setha T, Nealon J, Socheat D, Chantha N, Nathan MB. Community-based use of the larvivorous fish Poecilia reticulata to control the dengue vector Aedes aegypti in domestic water storage containers in rural Cambodia. J Vector Ecol. 2008;33:139-144.

18. Seng CM, Setha T, Nealon J, Chantha N, Socheat D, Nathan MB. The effect of long-lasting insecticidal water container covers on field populations of Aedes aegypti (L) mosquitoes in Cambodia. $J$ Vector Ecol. 2008;33:333-341. doi:10.3376/1081-1710-33.2.333.

19. Hustedt J, Doum D, Keo V, et al. Determining the efficacy of guppies and pyriproxyfen (Sumilarv ${ }^{\circledR}$ 2MR) combined with community engagement on dengue vectors in Cambodia: study protocol for a randomized controlled trial. Trials. 2017;18:367. doi:10.1186/s13063-017-2105-2.

20. Somboon P, Prapanthadara LA, Suwonkerd W. Insecticide susceptibility tests of Anopheles minimus s.1., Aedes aegypti, Aedes albopictus, and Culex quinquefasciatus in northern Thailand. Southeast Asian J Trop Med Public Health. 2003;34:87-93.

21. Rodríguez MM, Bisset JA, Fernández D. Levels of insecticide resistance and resistance mechanisms in Aedes aegypti from some Latin American countries. J Am Mosq Control Assoc. 2007;23:420-429.

22. Kamgang B, Marcombe S, Chandre F, et al. Insecticide susceptibility of Aedes aegypti and Aedes albopictus in Central Africa. Parasit Vectors. 2011;4:79. doi:10.1186/1756-3305-4-79.

23. Dusfour I, Zorrilla P, Guidez A, et al. Deltamethrin resistance mechanisms in Aedes aegypti populations from three French overseas territories worldwide. PLoS Negl Trop Dis. 2015;9:e0004226. doi:10.1371/journal.pntd.0004226.

24. Marcombe S, Mathieu RB, Pocquet N, et al. Insecticide resistance in the dengue vector Aedes aegypti from martinique: distribution, mechanisms and relations with environmental factors. PLoS One. 2012;7:e30989. doi:10.1371/journal.pone.0030989.

25. Faucon F, Dusfour I, Gaude T, et al. Identifying genomic changes associated with insecticide resistance in the dengue mosquito Aedes aegypti by deep targeted sequencing. Genome Res. 2015;25:1347-1359. doi:10.1101/gr.189225.115.

26. Prapanthadara L, Promtet N, Koottathep S, et al. Mechanisms of DDT and permethrin resistance in Aedes aegypti from Chiang Mai, Thailand. Dengue Bull. 2002;26:185-189.

27. Kawada H, Oo SZ, Thaung S, et al. Co-occurrence of point mutations in the voltage-gated sodium channel of pyrethroid-resistant Aedes aegypti populations in Myanmar. PLoS Negl Trop Dis. 2014;8:e3032. doi:10.1371/journal.pntd.0003032.

28. Stenhouse SA, Plernsub S, Yanola J, et al. Detection of the V1016G mutation in the voltage-gated sodium channel gene of Aedes aegypti (Diptera: Culicidae) by allele-specific PCR assay, and its distribution and effect on deltamethrin resistance in Thailand. Parasit Vectors. 2013;6:253. doi:10.1186/17563305-6-253.

29. Li CX, Kaufman PE, Xue RD, et al. Relationship between insecticide resistance and $k d r$ mutations in the dengue vector Aedes aegypti in Southern China. Parasit Vectors. 2015;8:325. doi:10.1186/s13071015-0933-z.

30. Miarinjara A, Boyer S. Current perspectives on plague vector control in Madagascar: susceptibility status of Xenopsylla cheopis to 12 insecticides. PLoS Negl Trop Dis. 2016;10:e004414. doi:10.1371/ journal.pntd.0004414. 